(C) Elsevier Sequoia S.A., Lausanne - Printed in the Netherlands

\title{
CALCULATION OF WEAR (f.i. wear modulus) IN THE PLASTIC CUP OF A HIP JOINT PROSTHESIS
}

\section{J. LIGTERINK}

Twente University of Technology, Enschede (The Netherlands)

(Received April 14, 1975)

\section{Summary}

The wear equation is applied to the wear process in a hip joint prosthesis and a wear modulus is defined. The sliding distance, wear modulus, wear volume, wear area, contact angle and the maximum normal stress were calculated and the theoretical calculations applied to test results.

During the wear process the increase of the wear modulus is about $100 \mathrm{Nmm}^{-2}$ per mm sliding distance in the Charnley and the CharnleyMuller hip joint prosthesis. From the wear volume point of view the Charnley prosthesis is probably superior to the Charnley-Muller prosthesis if run-in before implantation.

\section{Introduction to the problem}

A better understanding of the wear of a hip joint prosthesis is required, particularly of the plastic cup of the prosthesis. Results of measurements are available [1] and it is of interest to develop a theory to allow a better understanding of the wear process of the plastic cup. First the possibility of calculating a wear modulus in the cup at any moment during the wear process requires consideration then the calculation of the wear volume and the wear area if the maximum wear depth is known.

\section{Solution of the above-mentioned problems}

\subsection{Calculation of the wear modulus}

The wear equation $[2,3]$ can be written as follows:

$$
\frac{\Delta d_{n}}{\Delta s}=\frac{\sigma_{n}}{W}
$$

where $\Delta d_{n}$ is a small part of the wear depth $(\mathrm{mm})$ and is measured perpendicular to the sliding surface; $\Delta s$ is a small part of the sliding distance $(\mathrm{mm})$; $\sigma_{n}$ is the normal stress $\left(\mathrm{Nmm}^{-2}\right)$ on the sliding surface; $W$ is the wear modulus $\left(\mathrm{Nmm}^{-2}\right)$; (see Fig. 1). 


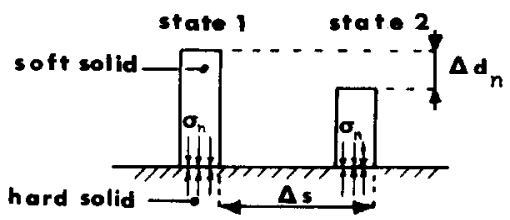

Fig. 1. Refers to eqn. (1).

$$
W=\frac{1}{K}
$$

where $K$ is the wear factor, mentioned in ref. 3 .

\section{Definition of a wear modulus}

The wear modulus is the normal stress on the sliding surface when the wear depth equals the sliding distance (see eqn. (1)).

Consider any point $P$ of the contact surface in the plastic cup during functioning of the prosthesis and assume that the magnitudes of the wear modulus at every point of the contact surface are equal to one another; see Figs. 2 and 3. Creep does not occur in the wear surface of the plastic cup [1] .

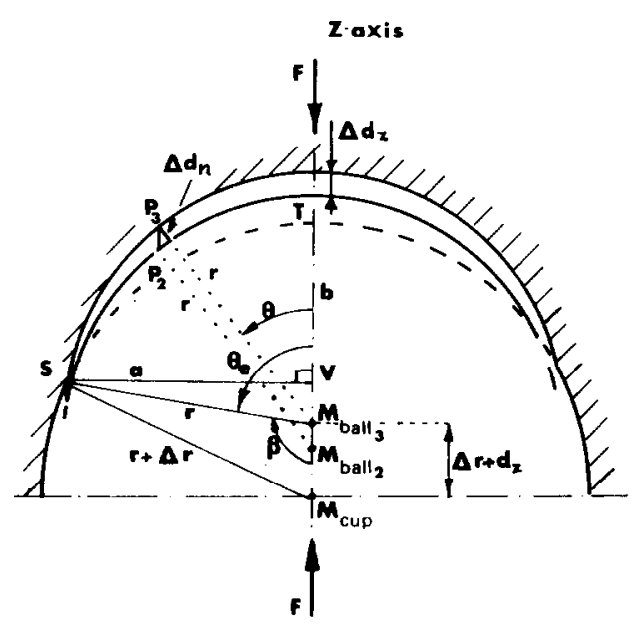

Fig. 2. Ball in cup.

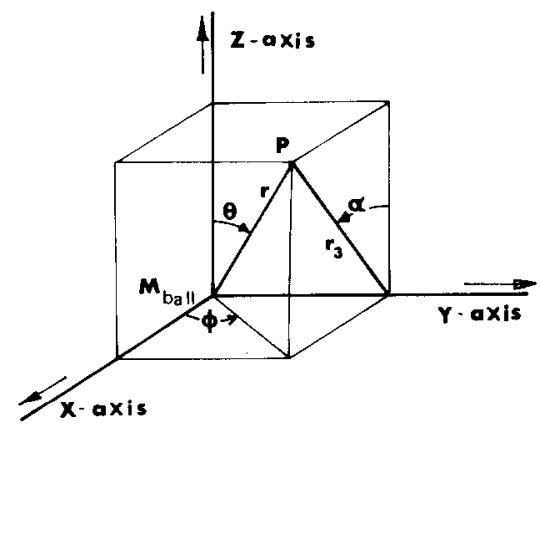

Fig. 3. System of coordinates.

Equation (1) is applied at point $P$ in Fig. 3 [7] where $M$ is the centre of the ball in the cup; $r$ is the radius $(\mathrm{mm})$ of the ball; $\theta$ is the angle (rad) between the radius $r$ and the $z$-axis (= the direction of the load $F(\mathrm{~N})$ on the ball and cup); $\Phi$ is the angle (rad) between the $x$-axis and the plane ${ } M_{\text {ball }}$ $z$-axis; the $y$-axis is the rotation axis of the ball of the prosthesis during functioning; $\alpha$ is the rotation angle (rad).

During the wear process the wear depth rates in the direction of the $z$-axis at every point of the contact surface are equal to one another $\left(=\Delta d_{z}\right)$; see Fig. 2: 


$$
P_{2} P_{3}=\Delta d_{z}=\frac{\Delta d_{n}}{\cos \theta}
$$

The sliding distance $(s)$ at point $P$, moves round the $y$-axis, see Fig. 3 :

$$
\begin{aligned}
& \Delta s=r_{3} \Delta \alpha \\
& \Delta \alpha=\omega \Delta t
\end{aligned}
$$

where $\omega$ is the angular speed $\left(\mathrm{rad} \mathrm{s}^{-1}\right)$ and $\Delta t$ is a small part of the time (s).

From Fig. 3:

$$
r_{3}=r \sqrt{\cos ^{2} \theta+\sin ^{2} \theta \cos ^{2} \Phi}
$$

Substituting eqns. (3), (4), (5) and (6) in eqn. (1) gives:

$$
\sigma_{n}=W \frac{\cos \theta}{\omega r \sqrt{\cos }^{2} \overline{\theta+\sin ^{2}} \bar{\theta}^{2} \cos ^{2} \bar{\Phi}} \frac{\Delta d_{z}}{\Delta t}
$$

When $\Phi=0$ :

$$
\sigma_{n \Phi=0}=\frac{W}{\omega r} \cos \theta \frac{\Delta d_{z}}{\Delta t}
$$

When $\Phi=90^{\circ}$ :

$$
\begin{aligned}
& \begin{aligned}
\sigma_{n \Phi=90^{\circ}} & =\frac{W \Delta \frac{\Delta d_{z}}{\omega r}}{\Delta t} \\
& =\sigma_{n \max }
\end{aligned} \\
& F=\int_{A=0}^{A}=\text { to tal contact area } \\
& \sigma_{z} \mathrm{~d} A
\end{aligned}
$$

where $F$ is the load on the ball (N); see Fig. $2 ; \sigma_{z}$ is the stress on the surface of the cup in the direction of the $z$-axis; $\mathrm{d} A$ is an infinitesimal area as function of $r, \theta$ and $\Phi$ :

$$
\begin{aligned}
& \mathrm{d} A=r^{2} \sin \theta \mathrm{d} \Phi \mathrm{d} \theta \\
& \sigma_{z}=\cos \theta \sigma_{n}
\end{aligned}
$$

Substituting eqns. (7), (9) and (10) in eqn. (8) gives

$$
F=\frac{4 r W}{\omega} \frac{\Delta d_{z}}{\Delta t} \int_{\Phi=0}^{\Phi=\frac{\pi}{2}} \int_{\theta=0}^{\theta=\theta_{e}} \frac{\cos ^{2} \theta \sin \theta}{{\sqrt{\cos ^{2}} \overline{\theta+\sin ^{2}} \overline{\theta \cos ^{2}}}^{2}} \mathrm{~d} \theta \mathrm{d} \Phi
$$

In eqn. (11):

$$
\frac{\cos ^{2} \theta \sin \theta}{{\sqrt{\cos ^{2}}}^{2} \overline{\theta+\sin }^{2} \overline{\theta \cos ^{2}} \bar{\Phi}}=G(\theta, \Phi)
$$

Numerical calculation of $G(\theta, \Phi)$ for the angles $0<\theta<\frac{\pi}{2}$ and $0<\Phi<\frac{\pi}{2}$
rad gives an approximation for $G(\theta, \Phi)$ :

$$
G(\theta, \Phi) \approx-0.81 \theta^{2}+1.17 \theta
$$

Substituting eqns. (12) and (13) in eqn. (11) gives: 


$$
\begin{aligned}
& F=\frac{4 r W}{\omega} \frac{\Delta d_{z}}{\Delta t}\left(0.92 \theta_{e}^{2}-0.424 \theta_{e}^{3}\right) \\
& W=\frac{\omega F}{4 r \frac{\Delta d_{z}}{\Delta t}\left(0.92 \theta_{e}^{2}-0.424 \theta_{e}^{3}\right)}
\end{aligned}
$$

where $\theta_{e}$ is the contact angle (rad) between the $z$-axis and the line $\mathbf{M}_{\mathrm{ball}} \mathrm{S}$ (see Fig. 2); $\mathrm{S}$ is a point on the edge of the contact area. Angle $\theta_{e}$ may be calculated, see Fig. 2 triangle: $\mathrm{M}_{\text {cup }} \mathrm{M}_{\mathrm{ball}} \mathrm{S}$ and page 68 of ref. 4 :

$$
\cos \beta=\frac{r^{2}+\left(\Delta r+d_{z}\right)^{2}-(r+\Delta r)^{2}}{2 r\left(\Delta r+d_{z}\right)}
$$

where $\Delta r$ is the radial clearance (mm) between ball and cup; $d_{z}$ is the maximum wear depth $(\mathrm{mm})$ in the direction of the $z$-axis.

$$
\cos \beta \approx \frac{-\Delta r}{\Delta r+d_{z}}
$$

and $\cos \theta_{e} \approx \frac{\Delta r}{\Delta r+d_{z}}$

\subsection{Calculation of the wear volume and the wear area}

The wear volume is determined by the difference of the volumes of the cones. The cones have a circular base of VS $=a \mathrm{~mm}$ radius; see Fig. 2; and a height of VT $=b \mathrm{~mm}$. The volume of a cone [4] is

$$
\begin{aligned}
& V_{\text {cone }}=\frac{\pi}{3} a^{2} b \\
& a=r \sin \theta_{e}
\end{aligned}
$$

From Fig. 2 and substituting eqn. (20) in eqn. (19) the wear volume is:

$$
V_{w}=\frac{\pi}{3} r^{2} \sin ^{2} \theta_{e} d_{z}
$$

Substituting eqn. (18) and $\sin ^{2} \theta_{e}+\cos ^{2} \theta_{e}=1$ in eqn. (21):

$$
V_{w}=\frac{\pi}{3} r^{2}\left(2 \Delta r+d_{z}\right) \frac{d_{z}^{2}}{\left(\Delta r+d_{z}\right)^{2}}
$$

The wear area can be calculated by the following formula [4], Fig. 2:

$$
\begin{aligned}
& A_{\text {ball }}=2 \pi r b \\
& b=r\left(1-\cos \theta_{e}\right)
\end{aligned}
$$

Substituting eqns. (18) and (24) in eqn. (23):

$$
A_{w}=2 \pi r^{2} \frac{d_{z}}{\Delta r+d_{z}}
$$

The maximum sliding distance in the prosthesis is equal to: 


$$
s=r \omega t
$$

where $r$ is the radius of the ball $(\mathrm{mm}) ; \omega$ is the angular speed ( $\left.\operatorname{rad~s}^{-1}\right) ; t$ is the time (s).

\section{Application of the solutions}

The experimental results of Paul et al. [1] are given in Table 1.

To calculate the sliding distance $(s)$, the wear modulus $(W)$, the wear volume $\left(V_{w}\right)$, the wear area $\left(A_{w}\right)$, the contact angle $(\theta)$ and the maximum stress in the prosthesis $\left(\sigma_{n \max }\right)$ the derived equations are used. One unknown factor $\Delta d_{z} / \Delta t$ in eqn. (15) can be obtained from Fig. 4 ; the points $\mathrm{C}_{1}, \mathrm{C}_{2}$, $\mathrm{CM}_{1}$ and $\mathrm{CM}_{2}$ being derived from Table 1 .

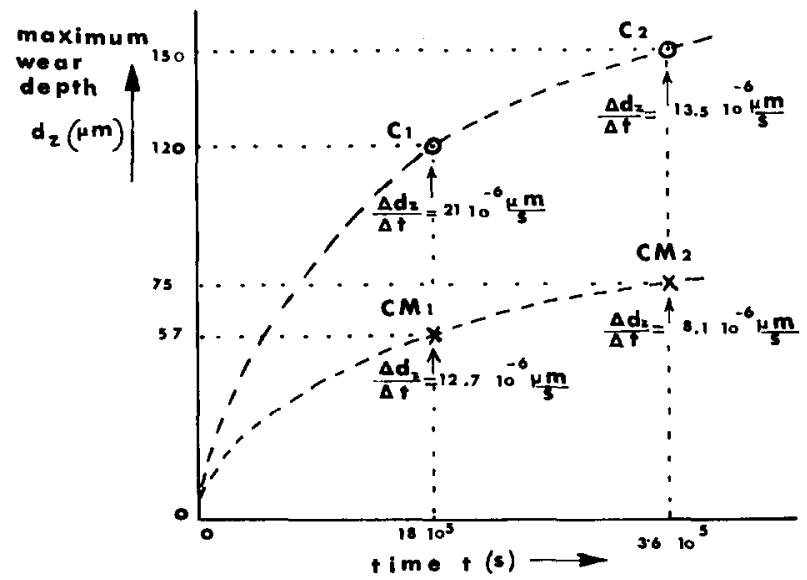

Fig. 4. Maximum wear depth as function of time in cup (see Table 1).

There are only a few test points available [1] concerning the wear depth $d_{z}$ as a function of time for the Charnley prosthesis: $\left(t=0 \mathrm{~s} ; d_{z}=\right.$ $0 \mu \mathrm{m}),\left(t=18 \times 10^{5} \mathrm{~s} ; d_{z}=120 \mu \mathrm{m}\right)$ and $\left(t=36 \times 10^{5} \mathrm{~s} ; d_{z}=150 \mu \mathrm{m}\right)$.

Similar data of the Charnley-Muller prosthesis are available. Suppose at the point ( $t=0 \mathrm{~s} ; d_{z}=0 \mu \mathrm{m}$ ) the required equations have roughly the following condition: $\Delta t / \Delta d_{z}=0$ or $\Delta d_{d} \Delta t=\infty$. A polynomial through the points $\mathrm{C}_{1}, \mathrm{C}_{2}$ and the points $\mathrm{CM}_{1}, \mathrm{CM}_{2}$ can now be calculated (see Fig. 4 and ref. 5). For the wear process in the Charnley prosthesis:

$$
t=0.938 d_{z}^{3}+0.00085 d_{z}^{4}
$$

For the wear process in the Charnley-Muller prosthesis:

$$
t=281 d_{z}^{2}+4.77 d_{z}^{3}
$$

where $t$ is the time (s) and $d_{z}$ is the maximum wear depth $(\mu \mathrm{m})$ in the cup.

The magnitudes of $\Delta d_{z} / \Delta t$ for the four points are given in Fig. 4.

Table 2 gives wear data calculated by the equations, Fig. 4 and Table 1 and

Fig. 5 is derived from this table. 


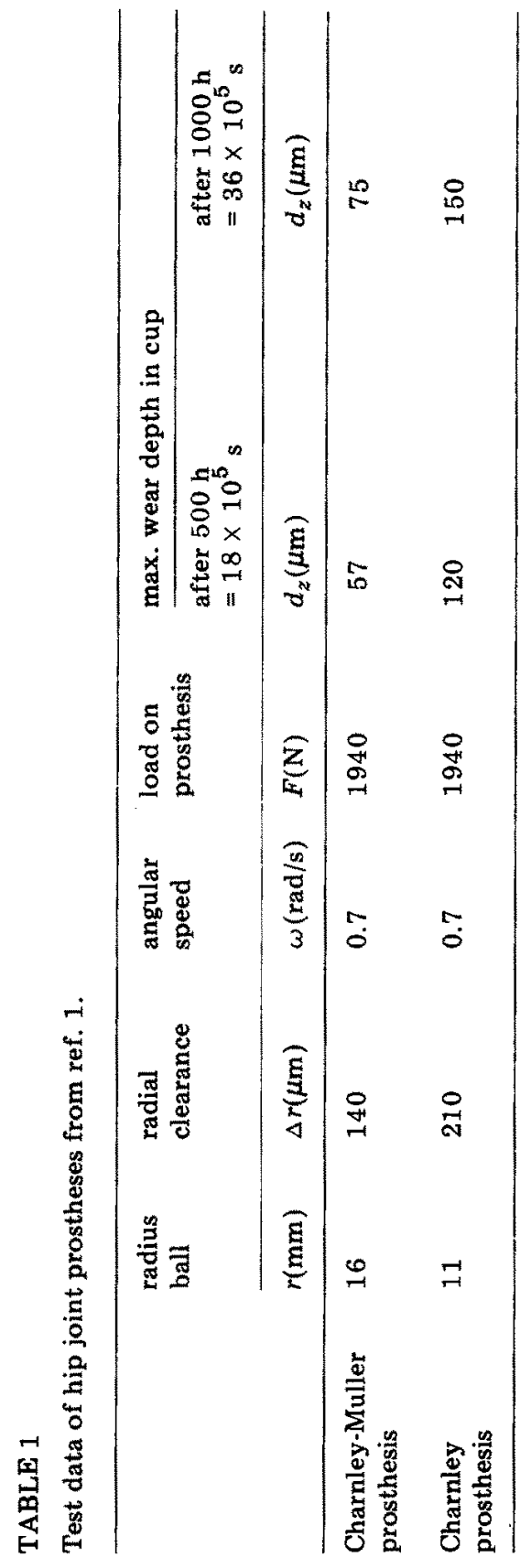




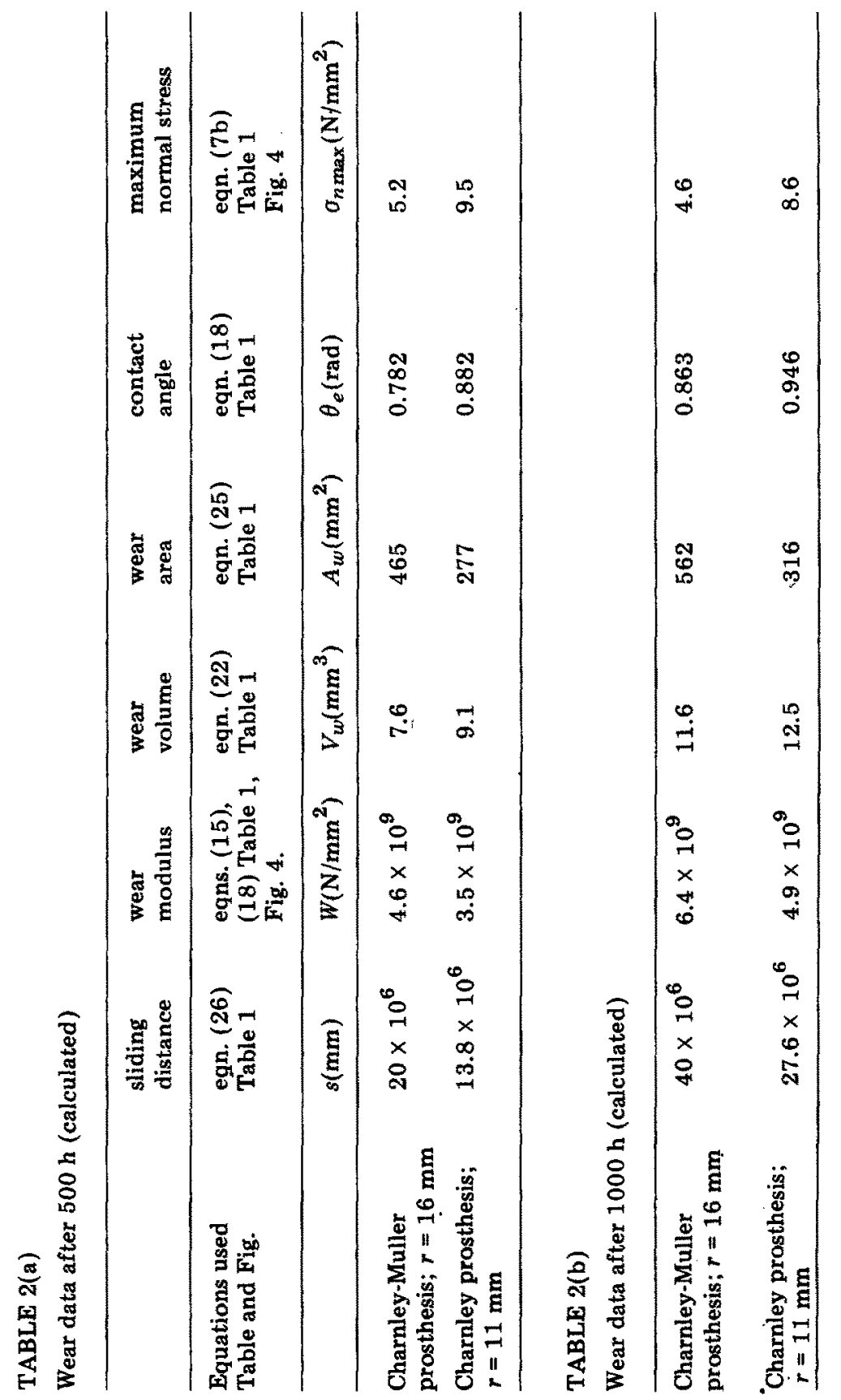




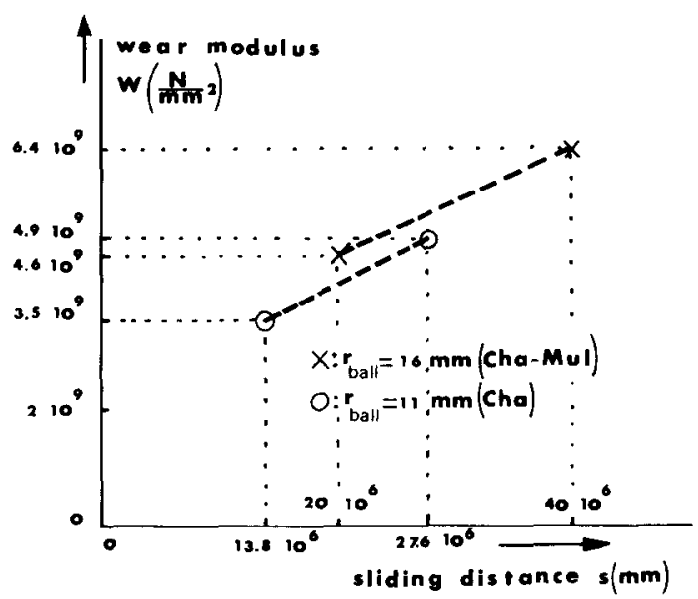

Fig. 5. Wear modulus as function of sliding distance in hip joint prostheses; derived from Table 2 (a) and Table $2(b)$; test conditions: vitallium against high density polyethylene, boundary lubricated, test is described in ref. 1 .

From Fig. 5 there is an increase of the wear modulus as a function of the sliding distance; this increase is equal to about $100 \mathrm{Nmm}^{-2}$ per $\mathrm{mm}$ sliding distance in both prostheses.

\section{Provisional conclusion}

(1) From 500 to $1000 \mathrm{~h}$ the magnitude of the wear volume for the Charnley-Muller prosthesis is: $4 \mathrm{~mm}^{3}$ and for the Charnley prostheses: 3.4 $\mathrm{mm}^{3}$.

(2) During the wear process the wear moduli in both prostheses change in the same way.

(3) The Charnley prosthesis is superior to the Charnley-Muller prosthesis if run-in before implantation if possible.

(4) The results confirm the conclusions of Charnley [6]

\section{Acknowledgements}

The author thanks the Tribology group of the Department of Mechanical Engineering for facilities provided and Miss J. Marree for assistance with the preparation of the manuscript.

\section{References}

1 B. O. Weightman, J. L. Paul, R. M. Rose, S. R. Simon and E. L. Radin, A coraparative study of total hip replacement prostheses, J. Biomechanics, 6 (1973) $299-311$.

2 J. F. Archard, Contact and rubbing flat surfaces, J. Appl. Phys., 24 (8) (1953) 981 - 988.

3 R. B. Lewis, Predicting the wear of sliding plastic surfaces, Mech. Eng., 86 (10) (1964) $32-35$.

4 F. Sass et al., Dubbels Taschenbuch für den Maschinenbau, Band I, Berlin, 1966. 
5 D. J. Ligterink, Beschrijving van meetresultaten met behulp van polynomen, Report WB74/TRI-6078, 1974-7-30, Twente University of Technology, Enschede.

6 J. Charnley, A. Kamagar and M. D. Longfield, The optimum size of prosthetic heads in relation to the wear of plastic sockets in total replacement of the hip, Med. Biol. Eng., 7 (1969) 31 - 39.

7 R. D. McLeish and J. Skorecki, Analysis of wear in a spherical joint. Proc. Instn. Mech. Engrs. 183 (1) (20) (1968 - 69) 389 - 392. 